\title{
Landau-Type Theory of Planar Crystal Plasticity
}

\author{
R. Baggio, ${ }^{1,2}$ E. Arbib, ${ }^{3}$ P. Biscari, ${ }^{3}$ S. Conti, ${ }^{4}$ L. Truskinovsky, ${ }^{2}$ G. Zanzotto, ${ }^{5}$ and O. U. Salman ${ }^{1}{ }^{1}$ \\ ${ }^{1}$ CNRS, LSPM, Université Paris 13, 93430 Villetaneuse, France \\ ${ }^{2}$ PMMH, ESPCI, F-75005 Paris, France \\ ${ }^{3}$ Department of Physics, Politecnico di Milano, 20133 Milano, Italy \\ ${ }^{4}$ Institut für Angewandte Mathematik, Universität Bonn, 53115 Bonn, Germany \\ ${ }^{5}$ DPG, Università di Padova, 35131 Padova, Italy
}

(Received 6 April 2019; revised manuscript received 28 September 2019; published 11 November 2019)

\begin{abstract}
We show that nonlinear continuum elasticity can be effective in modeling plastic flows in crystals if it is viewed as a Landau theory with an infinite number of equivalent energy wells whose configuration is dictated by the symmetry group $\operatorname{GL}(2, \mathbb{Z})$. Quasistatic loading can be then handled by athermal dynamics, while lattice-based discretization can play the role of regularization. As a proof of principle, we study dislocation nucleation in a homogeneously sheared $2 \mathrm{D}$ crystal and show that the global tensorial invariance of the elastic energy foments the development of complexity in the configuration of collectively nucleating defects. A crucial role in this process is played by the unstable higher symmetry crystallographic phases, typically thought to be unrelated to plastic flow.
\end{abstract}

DOI: 10.1103/PhysRevLett.123.205501

Crystal plasticity is the simplest among yield phenomena in solids [1], yet it has been compared in complexity to fluid turbulence [2,3]. The intrinsic irregularity of plastic flow in crystals [4] is due to short- and long-range interaction of crystal defects (dislocations) [5] dragged by the applied loading through a rugged energy landscape [6-8]. A fundamental understanding of plastic flow in crystals is crucial for improving hardening properties of materials [9], extending their fatigue life [10], controlling their forming at submicron scales [11,12], and building new materials [13].

Macroscopic crystal plasticity relies on a phenomenological continuum description of plastic deformation in terms of a finite number of order parameters representing amplitudes of predesigned mechanisms. These mechanisms are coupled elastically and operate according to friction-type dynamics [14-18]. The alternative microscopic approaches, relying instead on molecular dynamics [19-27], can handle only macroscopically insignificant time- and length scales [28]. An intermediate discrete dislocation dynamics approach focuses on long-range interaction of few dislocations, while their short-range interaction is still treated phenomenologically [29-31]. Collective dynamics of many dislocations can be also described by the dislocation density field; however, rigorous coarse graining in such a strongly interacting system still remains a major challenge [32-39].

A computational bridge between microscopic and macroscopic approaches is provided by the quasicontinuum finite element method, which uses adaptive meshing and employs $a b$ initio approaches to guide the constitutive response at different mesh scales [40-44]. Its drawbacks, however, are spurious effects due to matching of finite element representations at different scales and the high computational cost of reconstructing the constitutive response at the smallest scales [45]. Another important approach is the phase field method applied to dislocations, where plasticity is again modeled by a set of scalar order parameters which now evolve in an infinitely periodic energy landscape and are coupled to conventional linear [46-51] or nonlinear [52,53] elasticity. Here the challenge is to correctly account for the coupling between these order parameters, reproduce the underlying tensorial periodicity using a scalar framework, and select the appropriate internal length scales shaping the structure of the dislocation cores.

In this Letter we propose a synthetic approach dealing with macroscopic quantities such as stresses and strains, while accounting correctly for the exact symmetry of the crystal lattice. Our main assumption is that mesoscale material elements are exposed to the periodic energy landscape which resolves lattice-invariant strains including shears related to slip [54-56]; see Fig. 1. Our approach

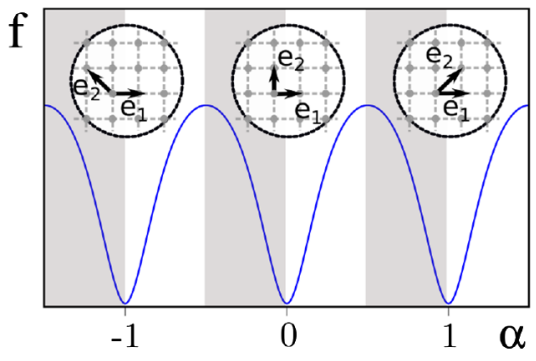

FIG. 1. Schematic representation of a lattice-invariant shear and the associated energy barriers along the simple shear loading path $\boldsymbol{\nabla} \mathbf{y}=\mathbb{1}+\alpha \mathbf{e}_{1} \otimes \mathbf{e}_{1}^{\perp}$. Alternating minimal-periodicity domains are marked in gray and white. 
follows the original proposal by Ericksen, that the energy periodicity in the space of tensors should be made compatible with geometrically nonlinear kinematics of crystal lattices [57-61], with the independent development of the mathematical formalism by Folkins [62]. This general program can be viewed as a far-reaching generalization of the Frenkel-Kontorova-Peierls-Nabarro model accounting for energy periodicity along a single slip plane [63-65]. Scalar models with periodic energies, dealing with multiple slip planes, have been used before to describe dislocation cores [66-68], to simulate dislocation nucleation [69-72], and to capture the intermittency of plastic flows $[6,73]$. Their tensorial versions with linearized kinematics were considered in Refs. [74-77].

In the proposed kinematically nonlinear $2 \mathrm{D}$ theory the role of the order parameter is played by the metric tensor (characterizing local deformation), and the bottoms of the energy wells correspond to lattice-invariant deformations. The continuum Landau energy density of this type must be invariant under the (infinite and discrete) symmetry group $\mathrm{GL}(2, \mathbb{Z})$ constituted by the $2 \times 2$ invertible matrices with integer entries. Since the ground state in this case is necessarily hydrostatic [58,78], regularization is necessary. Theories incorporating various elements of the tensorial $\mathrm{GL}(3, \mathbb{Z})$ symmetry have already proved useful in the description of reconstructive phase transitions [56,79-85], and in this Letter we extend this idea to the modeling of crystal plasticity proper.

From the perspective of our Landau-type theory with an infinite number of equivalent energy wells, a plastically deformed solid can be viewed as a mixture of equivalent phases which are related by a large "transformation strain" responsible for localization. Elastically compatible parts of the boundaries between such phases have no energy cost and are therefore invisible. The incompatible parts are represented by dislocations, which generate long-range elastic strain fields. Plastic yield can then be interpreted as an escape from the reference energy well and plastic mechanisms can be linked to low-barrier valleys in the energy landscape. Friction-type dissipation emerges as a result of homogenization of an overdamped athermal dynamics in a rugged energy landscape [86,87]. It is important to notice that such an approach incorporates both long- and short-range dislocation interactions; it also correctly describes plastic slip even though the dislocations cores are regularized and blurred on the scale of the unit cell.

In this Letter we use this general approach to study the peculiarities of collective dislocation nucleation in crystals with different symmetries and show that there is a nontrivial coupling between plastic mechanisms due to the presence of degenerate mountain passes representing lattices with higher symmetry. The crucial role in the formation of selfinduced plastic disorder is then played by the unstable high symmetry phases, conventionally thought to be

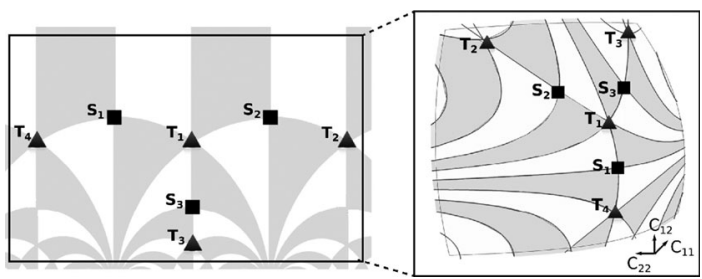

FIG. 2. Structure of the $\operatorname{GL}(2, \mathbb{Z})$ periodicity domains in the space of metric tensors. (a) Partition of the complex half plane (Dedekind tessellation). (b) Equivalent partition of the section $\operatorname{det} \mathbf{C}=1$ of the space $\mathbf{C}$. Points $\mathbf{S}_{\mathbf{i}}$ represent the same square lattice, and points $\mathbf{T}_{\mathbf{i}}$ the same triangular lattice.

unrelated to plastic flow. The general conclusion is that the global crystal symmetry can induce frustration and become the cause of lattice incompatibility developing during plastic deformation.

Consider a continuous deformation $\mathbf{y}=\mathbf{y}(\mathbf{x})$, where $\mathbf{y}$ are actual and $\mathbf{x}$ are reference coordinates. The energy density of an elastic solid can depend on the deformation gradient $\boldsymbol{\nabla} \mathbf{y}$ only through the metric tensor $\mathbf{C}=(\nabla \mathbf{y})^{T} \boldsymbol{\nabla} \mathbf{y}$. To account for all deformations that map a Bravais lattice onto itself, we require that the strain energy density $f(\mathbf{C})$ equals $f\left(\mathbf{m}^{\mathbf{T}} \mathbf{C m}\right)$ for any $\mathbf{m}$ in $\operatorname{GL}(2, \mathbb{Z})$; see [88]. In the presence of such symmetry, the space of metric tensors $\mathbf{C}$ partitions into periodicity domains, with each one containing an energy well equivalent to the reference one. If we know the structure of the energy in one such domain, we can use, for instance, the Lagrange reduction $[85,89]$ to find its value at any other point. In the special case of $2 \mathrm{D}$ lattices, which we focus on here, a section $\operatorname{det} \mathbf{C}=1$ in the 3D space of tensors $\mathbf{C}$ can be used to visualize the implied tensorial periodicity of the energy; see Fig. 2. The global picture is made visible if we map this section onto a complex half plane using the function $z=C_{11}^{-1}\left(C_{12}+i\right)$ $[61,62]$. For instance, the point $S_{1}$ in Fig. 2, corresponding on the complex half plane to $z=i$ describes a square lattice with the basis vectors aligned with the closepacked directions: $\mathbf{e}_{1}=(1,0), \mathbf{e}_{2}=(0,1)$. Simple shear $\mathbb{1}+\mathbf{e}_{1} \otimes \mathbf{e}_{1}^{\perp}$, where $\mathbf{e}_{1}^{\perp}$ is a unit vector orthogonal to $\mathbf{e}_{1}$, maps this point onto its symmetric counterpart $i+1$ (point $\mathbf{S}_{\mathbf{2}}$ ); another square lattice, corresponding to point $\mathbf{S}_{\mathbf{3}}$ in Fig. 2 with $z=\frac{1}{2}(1+i)$, can be obtained from the lattice $\mathbf{S}_{\mathbf{1}}$ by the shear $\mathbb{1}+\mathbf{e}_{2} \otimes \mathbf{e}_{2}^{\perp}$. Instead, the point $\mathbf{T}_{\mathbf{1}}$ in Fig. 2, corresponding to $z=\frac{1}{2}+(\sqrt{3} / 2) i$, describes a triangular lattice (with hexagonal symmetry) whose basis vectors $\mathbf{e}_{1}=\gamma(1,0), \mathbf{e}_{2}=\gamma(1 / 2, \sqrt{3} / 2)$ with $\gamma=(4 / 3)^{1 / 4}$ are again aligned with the close-packed directions. Its closest equivalent neighbors are $\mathbf{T}_{\mathbf{2}}$ and $\mathbf{T}_{\mathbf{4}}$, corresponding to $z=\frac{1}{2}+(\sqrt{3} / 2) i \pm 1$. They are reachable from $\mathbf{T}_{\mathbf{1}}$ by the shear deformations $\mathbb{1} \pm \mathbf{e}_{1} \otimes \mathbf{e}_{1}^{\perp}$.

To demonstrate the possibility of yield-inducing instabilities in a material with such energy, it is sufficient to consider a system under the most constraining affine 
displacement control. Given the gradient nature of the order parameter, the (spinodal) instability of a homogeneous state should be linked to the local loss of rank-1 convexity of the energy $[90,91]$. This is equivalent to the loss of positive definiteness of the acoustic tensor $\mathbf{Q}$ with components $Q_{i k}=A_{i j k l} n_{j} n_{l}$, where $A_{i j k l}=\partial^{2} f /\left(\partial_{j} y_{i} \partial_{l} y_{k}\right)$ is the fourth-order incremental elastic tensor, and $\mathbf{n}$ is a unit vector $[92,93]$.

For illustrative purposes we now choose a particular energy density $f=f_{v}+f_{d}$ which decouples into a volumetric $f_{v}(\operatorname{det} \mathbf{C})$ and a distortive $f_{d}\left[\mathbf{C} /(\operatorname{det} \mathbf{C})^{1 / 2}\right]$ part. Since $\operatorname{det} \mathbf{C}$ is invariant under $\operatorname{GL}(2, \mathbb{Z})$, our symmetry constraints concern only the deviatoric part $f_{d}$. This function needs to be specified only inside a single periodicity domain, with the suitable conditions on its boundary ensuring required smoothness [61,94]. The lowest-order polynomial representation of $f_{d}$, which guarantees the continuity of the elastic moduli, was constructed in Ref. [85]; for the general nonpolynomial representation see Ref. [62].

If the reference lattice is either square or triangular, the minimal potential can be chosen in the form [85]

$$
f_{d}(\tilde{\mathbf{C}})=\beta \psi_{1}(\tilde{\mathbf{C}})+\psi_{2}(\tilde{\mathbf{C}}), \tilde{\mathbf{C}}=\mathbf{C} /(\operatorname{det} \mathbf{C})^{1 / 2}
$$

where $\psi_{1}=I_{1}{ }^{4} I_{2}-41 I_{2}{ }^{3} / 99+7 I_{1} I_{2} I_{3} / 66+I_{3}{ }^{2} / 1056$, and $\psi_{2}=4 I_{2}{ }^{3} / 11+I_{1}{ }^{3} I_{3}-8 I_{1} I_{2} I_{3} / 11+17 I_{3}{ }^{2} / 528$. The hexagonal invariants here are $I_{1}=\frac{1}{3}\left(\tilde{C}_{11}+\tilde{C}_{22}-\tilde{C}_{12}\right)$, $I_{2}=\frac{1}{4}\left(\tilde{C}_{11}-\tilde{C}_{22}\right)^{2}+\frac{1}{12}\left(\tilde{C}_{11}+\tilde{C}_{22}-4 \tilde{C}_{12}\right)^{2}$, and $I_{3}=$ $\left(\tilde{C}_{11}-\tilde{C}_{22}\right)^{2}\left(\tilde{C}_{11}+\tilde{C}_{22}-4 \tilde{C}_{12}\right)-\frac{1}{9}\left(\tilde{C}_{11}+\tilde{C}_{22}-4 \tilde{C}_{12}\right)^{3}$. The choice $\beta=-1 / 4$ enforces the square symmetry on the reference state, while, choosing $\beta=4$, we bias the reference state toward hexagonal symmetry; the energy landscapes in those two cases are illustrated in Fig. 3. The volumetric energy density will be chosen in the simplest form $f_{v}(s)=\mu(s-\log s)$, which excludes configurations with infinite compression; the coefficient $\mu$ plays the role of a bulk modulus.

The resulting "yield surfaces" are shown in Fig. 3. To understand the nature of the associated instabilities,

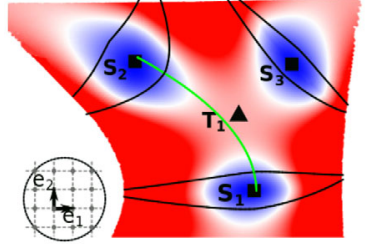

(a)

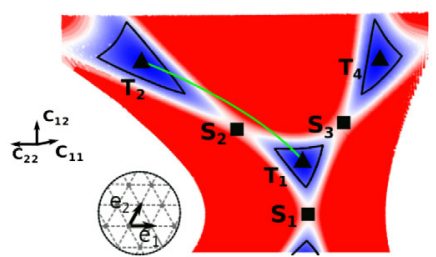

(b)
FIG. 3. Energy landscapes corresponding to potential (1) with (a) $\beta=-1 / 4$ and (b) $\beta=4$. Colors indicate the energy level: blue, low; red, high. Black lines delimit the zones of linear stability for the homogeneous states. Green lines correspond to the simple shear loading paths discussed in the text. consider the case in which a simple shear is imposed on the boundary

$$
\boldsymbol{\nabla} \mathbf{y}=\mathbb{1}+\alpha \mathbf{e}_{1} \otimes \mathbf{e}_{1}^{\perp}
$$

Starting, in the case of square lattice, with the homogeneous reference state $\mathbf{S}_{\mathbf{1}}$, we find that at instability point the condition $\operatorname{det} \mathbf{Q}=0$ produces two almost simultaneously destabilized directions $\mathbf{q}=\boldsymbol{\nabla y}[\mathbf{n}] /|\nabla \mathbf{y}[\mathbf{n}]|=$ $(\cos \xi, \sin \xi)$ : the first one with $\xi \approx-0.11 \mathrm{rad}$, almost perpendicular to the deformed $\mathbf{e}_{2}$, and the second one with $\xi \approx 1.55 \mathrm{rad}$, almost perpendicular to the deformed $\mathbf{e}_{1}$. The near degeneracy of the bifurcation is an indicator that two "slip planes" may be activated. In the case of a triangular lattice, the instability along a similar loading path originating at $\mathbf{T}_{\mathbf{1}}$ produces a single unstable direction $\xi \approx$ $-1.25 \mathrm{rad}$ which is incommensurate with the lattice. In this case one can expect only one slip plane to be activated. Our numerical experiments show that the acoustic-tensorbased analytical instability conditions are in agreement with direct numerical simulations.

Consider now a single edge dislocation trapped by the lattice far from the boundaries. In Fig. 4 we illustrate the corresponding stress distribution which matches the classical continuum far field with $r^{-1}$ asymptotics while also resolving (at a scale of the mesh) the core region. Matching the structure of the dislocation core with the results of quantum mechanical simulations can be used to calibrate the model parameters $[95,96]$. In particular, our symmetry requirements on the potential leave ample room for tuning the quantities like the dislocation energy and the Peierls stress.

The collective nucleation pattern emerging after a stress drop is illustrated in Fig. 5 for both types of lattices. The results are presented in both the configurational spaceFigs. 5(a) and 5(c), where each point corresponds to a single element of the mesh-and the actual physical space-Figs. 5(b) and 5(d), where the color of the nodal points indicates the level of stress. The configurational points, all located initially at the bottom of the reference
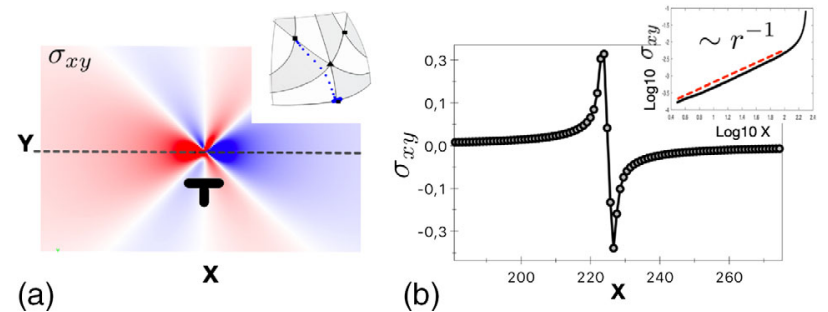

FIG. 4. Single edge dislocation in a square lattice linking the wells $\mathbf{S}_{\mathbf{1}}$ and $\mathbf{S}_{\mathbf{2}}$; its Burgers vector is horizontal, with the length equal to the side of the square unit cell. (a) Finite element nodes with coloring indicating the level of Cauchy stress $\sigma_{x y}$. (Inset) Image of this defect in the configurational space. (b) Stress profile along the glide plane. (Inset) The far field asymptotics. System size, $1000 \times 1000$. 

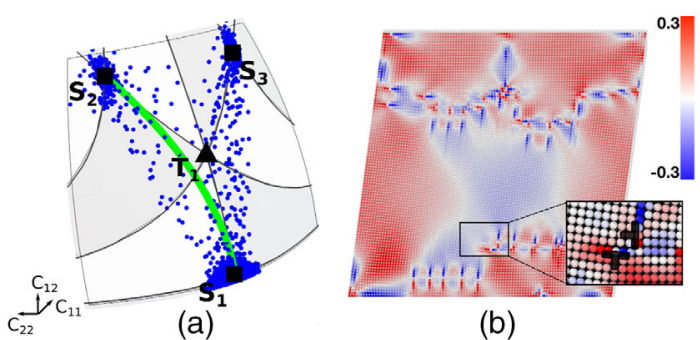

(b)

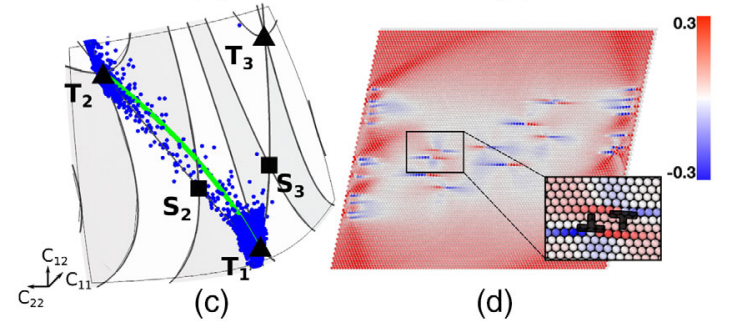

FIG. 5. Collective dislocation nucleation. (a),(b) Square lattice. (c),(d) Triangular lattice. We show two representations of the same phenomenon. (a),(c) In the configurational space, where green lines are simple shear paths imposed by the loading device and blue dots indicate the metric tensor distribution among the elements. (b),(d) In the physical space. Colors indicate the level of the nodal Cauchy stresses $\sigma_{x y}$. System size, $200 \times 200$.

energy well, disperse as a result of the massive nucleation event. The ensuing spatial dislocation distribution is quasiregular, with pileups at the rigid boundaries. Note the formation of characteristic entanglements with dislocations on two slip planes blocking each other (in the case of a square lattice).

Note that in Fig. 5(a) the square lattice is driven by the loading device from the reference state $\mathbf{S}_{1}$ toward the equivalent state $\mathbf{S}_{\mathbf{2}}$. At the "yielding" threshold, which marks the end of the elastic regime, the homogeneous configuration $\mathbf{S}_{\mathbf{1}}$ loses stability and the ensuing pattern represents (outside the core regions) a mixture of three "pure" states, $\mathbf{S}_{\mathbf{1}}, \mathbf{S}_{\mathbf{2}}$, and $\mathbf{S}_{\mathbf{3}}$. While the appearance of state $\mathbf{S}_{\mathbf{2}}$ is natural because the corresponding "plastic mechanism" is favored by the loading, the main complexity of the resulting dislocation pattern is due to the emergence of state $\mathbf{S}_{3}$. This indicates the activation of the second plastic mechanism, decoupled (in the nonlinear theory) from the first one.

The appearance of state $\mathbf{S}_{\mathbf{3}}$ can be understood if we recall that the linear stability analysis predicts two almost simultaneously unstable modes aligned with the slip directions in the deformed state. While one of these directions is indeed aiming toward the energy well $\mathbf{S}_{\mathbf{2}}$, the other one, which bifurcates first, is directed toward $\mathbf{S}_{\mathbf{3}}$. Our numerical simulations show that the latter instability mode grows faster, which can be interpreted, somewhat counterintuitively, as early stage dominance of the secondary plastic mechanism. The flow of configurational points passes near the unstable equilibrium state $\mathbf{T}_{1}$, corresponding to a triangular lattice, where it splits into three streams directed toward the configurations $\mathbf{S}_{\mathbf{1}}, \mathbf{S}_{\mathbf{2}}$, and $\mathbf{S}_{\mathbf{3}}$; see Movie S1 in the Supplemental Material [88].

This behavior becomes more transparent if we consider a smoother energy potential. Note that the symmetry transformations from $\mathrm{GL}(2, \mathbb{Z})$ correspond on the upper complex half plane to the fractional (Möbius) transformations with integral entries of the type $\left(m_{22} z+m_{12}\right) /\left(m_{21} z+m_{11}\right)$ $[62,94]$. This observation links $[61,62]$ the infinitely periodic energy densities for 2D crystalline materials with the classical modular functions [97], with the most wellknown example provided by the Klein invariant $J(z)$ [98]; see the Supplemental Material for more details [88]. One can show that, for this holomorphic function $\left.J\right|_{\mathbf{S}_{\mathbf{i}}}=1$, $\left.J^{\prime}\right|_{\mathbf{S}_{\mathbf{i}}}=0$, while $\left.J\right|_{\mathbf{T}_{\mathbf{i}}}=\left.J^{\prime}\right|_{\mathbf{T}_{\mathbf{i}}}=\left.J^{\prime \prime}\right|_{\mathbf{T}_{\mathbf{i}}}=0$. Therefore, the corresponding potentials with the reference square and triangular lattices can be chosen in the form $f_{d}(z)=$ $|J(z)-1|$ (square lattice) and $f_{d}(z)=|J(z)|^{2 / 3}$ (triangular lattice); the exponents are chosen to ensure a nondegenerate linear-elastic response close to the bottoms of the energy wells. The energy landscapes and the yield surfaces for such potentials are qualitatively similar to the ones presented in Fig. 3.

Note that the choice $f_{d}(z)=|J(z)-1|$ for a square lattice turns the triangular critical point $\mathbf{T}_{\mathbf{1}}$ and all of its symmetric counterparts into degenerate "monkey saddles," characterized by the local Taylor expansion of the form $x^{3}-3 x y^{2}$; see Fig. 6(a) and also [99]. The flow of configurational points directed initially toward such an unstable state (say, $\mathbf{T}_{\mathbf{1}}$ ) will therefore necessarily split into three streams directed toward the stable states (say, $\mathbf{S}_{\mathbf{1}}, \mathbf{S}_{\mathbf{2}}$, and $\mathbf{S}_{\mathbf{3}}$ ).

The situation looks a bit different in the case of the polynomial energy (1), where the Hessian is nondegenerate at the point $\mathbf{T}_{\mathbf{1}}$, which corresponds in this case to a shallow energy maximum. However, this maximum is surrounded by the three nondegenerate saddles $\mathbf{R}_{\mathbf{1}}, \mathbf{R}_{\mathbf{2}}$, and $\mathbf{R}_{\mathbf{3}}$ describing rhombic lattices [see Fig. 6(b)], and the general conclusion about the activation of the secondary plastic mechanism and the ultimate dispersion over the three energy wells $\mathbf{S}_{\mathbf{1}}, \mathbf{S}_{\mathbf{2}}$, and $\mathbf{S}_{\mathbf{3}}$ remains valid. Note that the implied coupling of the plastic mechanisms would have to
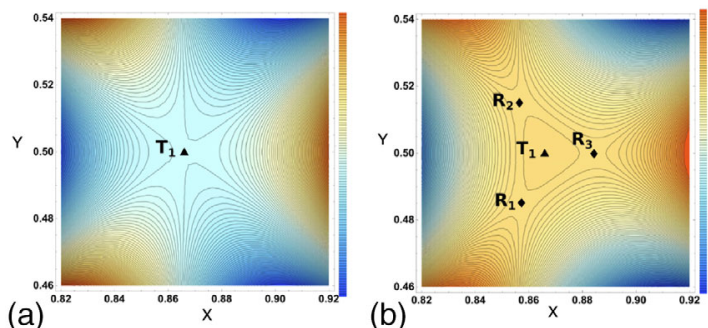

FIG. 6. Level sets of the energy density on the surface $\operatorname{det} \mathbf{C}=1$ around the point $\mathbf{T}_{\mathbf{1}}$. We use the parametrization $C_{11}=1 / y$, $C_{22}=\left(x^{2}+y^{2}\right) / y, C_{12}=x / y$. (a) Klein-invariant-based potential. (b) Polynomial potential (1) with $\beta=-1 / 4$. 
be postulated in the phenomenological plasticity theory [100-102]; however, it can also be reconstructed from ab initio calculations [99].

The picture is simpler in the case of a triangular lattice where the loading (2) from $\mathbf{T}_{\mathbf{1}}$ to $\mathbf{T}_{\mathbf{2}}$ produces a mixture of only two pure states, $\mathbf{T}_{1}, \mathbf{T}_{\mathbf{2}}$; see Fig. 5(d). The latter can be interpreted as the activation of a single plastic mechanism, the one favored by the loading; see Movie S2 in the Supplemental Material [88].

To conclude, our model shows that crystal plasticity naturally arises from nonlinear elasticity if the tensorial symmetry of the crystal lattice is properly accounted for. Athermal evolution in the regularized model of this type can lead to temporal and spatial complexity, which our analysis predicts to be highly sensitive to both the crystallographic symmetry and the orientation of the crystal $[103,104]$. The study of a $2 \mathrm{D}$ version of the theory highlights the crucial role played in plastic deformation by the degenerate saddle points of the energy [99] representing seemingly irrelevant, unstable crystallographic phases; for similar effects in other fields see Refs. [105-108]. The development of the potentials with $\mathrm{GL}(3, \mathbb{Z})$ symmetry will be necessary to account for cross slip, stacking faults, Frank-Read sources, and other crucial features of 3D crystal plasticity.

The work was partially supported by the grant ANR-17-CE08-0047 (O. U.S. and L. T.) and the Deutsche Forschungsgemeinschaft (DFG) via Project No. 211504053-SFB 1060 (S. C.).

[1] D. Bonn, M. M. Denn, L. Berthier, T. Divoux, and S. Manneville, Rev. Mod. Phys. 89, 035005 (2017).

[2] A. H. Cottrell, in Dislocations in Solids, edited by F. R. N. Nabarro and M. S. Duesbery, Vol. 11 (Elsevier, New York, 2002), pp. vii-Xvii.

[3] W. Choi, Y. Chen, S. Papanikolaou, and J. Sethna, Comput. Sci. Eng. 14, 33 (2012).

[4] A. Vinogradov and Y. Estrin, Prog. Mater. Sci. 95, 172 (2018).

[5] L. A. Zepeda-Ruiz, A. Stukowski, T. Oppelstrup, and V. V. Bulatov, Nature (London) 550, 492 (2017).

[6] O. Salman and L. Truskinovsky, Int. J. Eng. Sci. (Oxford) 59, 219 (2012).

[7] L. Kubin, Dislocations, Mesoscale Simulations and Plastic Flow, Vol. 5 (Oxford University Press, New York, 2013).

[8] P. D. Ispánovity, L. Laurson, M. Zaiser, I. Groma, S. Zapperi, and M. J. Alava, Phys. Rev. Lett. 112, 235501 (2014).

[9] D. A. Hughes and N. Hansen, Acta Mater. 148, 374 (2018).

[10] A. Irastorza-Landa, H. Van Swygenhoven, S. Van Petegem, N. Grilli, A. Bollhalder, S. Brandstetter, and D. Grolimund, Acta Mater. 112, 184 (2016).

[11] F. F. Csikor, C. Motz, D. Weygand, M. Zaiser, and S. Zapperi, Science 318, 251 (2007).

[12] P. Zhang, O. U. Salman, J.-Y. Zhang, G. Liu, J. Weiss, L. Truskinovsky, and J. Sun, Acta Mater. 128, 351 (2017).
[13] D. L. McDowell, in Computational Materials System Design, edited by D. Shin and J. Saal (Springer, Cham, 2018), pp. 105-146.

[14] F. Roters, P. Eisenlohr, L. Hantcherli, D. D. Tjahjanto, T. R. Bieler, and D. Raabe, Acta Mater. 58, 1152 (2010).

[15] S. Forest, J. R. Mayeur, and D. L. McDowell, Micromorphic crystal plasticity, in Handbook of Nonlocal Continuum Mechanics for Materials and Structures, edited by G. Z. Voyiadjis (Springer, Cham, 2019), pp. 643-686.

[16] M. E. Gurtin and L. Anand, J. Mech. Phys. Solids 57, 405 (2009).

[17] C. S. Han, H. Gao, Y. Huang, and W. D. Nix, J. Mech. Phys. Solids 53, 1188 (2005).

[18] Encyclopedia of Computational Mechanics, 2nd ed., Vol. 44, CISM Courses and Lectures Vol. 292, edited by E. Stein, R. de Borst, and T. J. R. Hughes (John Wiley \& Sons, Chichester, England, 2018), pp. 1-23.

[19] Y.-M. Juan and E. Kaxiras, J. Comput.-Aided Mater. Des. 1, 55 (1993).

[20] V. Bulatov, F. F. Abraham, L. Kubin, B. Devincre, and S. Yip, Nature (London) 391, 669 (1998).

[21] D. Lyu and S. Li, J. Mech. Phys. Solids 122, 613 (2019).

[22] D. L. McDowell, in Handbook of Materials Modeling: Methods: Theory and Modeling, edited by W. Andreoni and S. Yip (Springer, Cham, 2018), pp. 1-21.

[23] P. Moretti, B. Cerruti, and M.-C. Miguel, PLoS One 6, e20418 (2011).

[24] K. R. Elder, M. Katakowski, M. Haataja, and M. Grant, Phys. Rev. Lett. 88, 245701 (2002).

[25] J. M. Tarp, L. Angheluta, J. Mathiesen, and N. Goldenfeld, Phys. Rev. Lett. 113, 265503 (2014).

[26] A. Skaugen, L. Angheluta, and J. Viñals, Phys. Rev. Lett. 121, 255501 (2018).

[27] C. Baruffi, A. Finel, Y. Le Bouar, B. Bacroix, and O. U. Salman, Mater. Theor. 3, 4 (2019).

[28] D. L. McDowell, in Mesoscale Models: From Micro-Physics to Macro-Interpretation, edited by S. Mesarovic, S. Forest, and H. Zbib (Springer, Cham, 2019), pp. 195-297.

[29] L. E. Shilkrot, R. E. Miller, and W. A. Curtin, J. Mech. Phys. Solids 52, 755 (2004).

[30] H. Song, H. Yavas, E. Van der Giessen, and S. Papanikolaou, J. Mech. Phys. Solids 123, 332 (2019).

[31] J. A. El-Awady, H. Fan, and A. M. Hussein, in Multiscale Materials Modeling for Nanomechanics, edited by C. R. Weinberger and G. J. Tucker, Springer Series in Materials Science, Vol. 245 (Springer, New York, 2016), p. 337.

[32] A. El-Azab, Phys. Rev. B 61, 11956 (2000).

[33] S. Xia and A. El-Azab, Model. Simul. Mater. Sci. Eng. 23, 055009 (2015).

[34] I. Groma, in Mesoscale Models: From Micro-Physics to Macro-Interpretation, edited by S. Mesarovic, S. Forest, and H. Zbib (Springer, Cham, 2019), pp. 87-139.

[35] A. Acharya and A. Roy, J. Mech. Phys. Solids 54, 1687 (2006).

[36] Y. S. Chen, W. Choi, S. Papanikolaou, M. Bierbaum, and J. P. Sethna, Int. J. Plast. 46, 94 (2013).

[37] S. Sandfeld, T. Hochrainer, M. Zaiser, and P. Gumbsch, J. Mater. Res. 26, 623 (2011). 
[38] R. LeSar, Annu. Rev. Condens. Matter Phys. 5, 375 (2014).

[39] P.-L. Valdenaire, Y. Le Bouar, B. Appolaire, and A. Finel, Phys. Rev. B 93, 214111 (2016).

[40] E. B. Tadmor, M. Ortiz, and R. Phillips, Philos. Mag. A 73, 1529 (1996).

[41] E. B. Tadmor and R. E. Miller, Model. Simul. Mater. Sci. Eng. 25, 071001 (2017).

[42] D. M. Kochmann and J.S. Amelang, in Multiscale Materials Modeling for Nanomechanics, edited by C. R. Weinberger and G. J. Tucker (Springer, Cham, 2016), pp. 159-193.

[43] J. Li, K. J. Van Vliet, T. Zhu, S. Yip, and S. Suresh, Nature (London) 418, 307 (2002).

[44] J. Li, T. Zhu, S. Yip, K. J. Van Vliet, and S. Suresh, Mater. Sci. Eng. A 365, 25 (2004).

[45] M. Luskin and C. Ortner, Acta Numer. 22, 397 (2013).

[46] A. Finel and D. Rodney, Phase field method and dislocations, in Influences of Interference and Dislocation Behavior on Microstructure Evolution, MRS Proceedings, edited by M. Aindow, M. Asta, M. Glazov, D. Medlin, A. Rollet, and M. Zaiser (Cambridge University Press, Cambridge, England, 2000), Vol. 65.

[47] Y. U. Wang, Y. M. Jin, A. M. Cuitiño, and A. G. Khachaturyan, Appl. Phys. Lett. 78, 2324 (2001).

[48] D. Rodney, Y. Le Bouar, and A. Finel, Acta Mater. 51, 17 (2003).

[49] C. Shen and Y. Wang, Acta Mater. 52, 683 (2004).

[50] A. Hunter, I. J. Beyerlein, T. C. Germann, and M. Koslowski, Phys. Rev. B 84, 144108 (2011).

[51] A. Ruffini, Y. Le Bouar, and A. Finel, J. Mech. Phys. Solids 105, 95 (2017).

[52] P. Biscari, M. F. Urbano, A. Zanzottera, and G. Zanzotto, J. Elast. 123, 85 (2016).

[53] M. Javanbakht and V. I. Levitas, Int. J. Solids Struct. 82, 95 (2016).

[54] V. Bulatov and A. Argon, Model. Simul. Mater. Sci. Eng. 2, 167 (1994).

[55] E. Kaxiras and L. L. Boyer, Phys. Rev. B 50, 1535 (1994).

[56] M. Pitteri and G. Zanzotto, Continuum Models for Phase Transitions and Twinning in Crystals (Chapman and Hall, London, 2002).

[57] J. L. Ericksen, Int. J. Solids Struct. 6, 951 (1970).

[58] J. L. Ericksen, in Nonlinear Elasticity, edited by R. W. Dickey (Academic Press, New York, 1973), pp. 161-173.

[59] J.L. Ericksen, in Advances in Applied Mechanics, Vol. 17, edited by C.-S. Yih (Elsevier, New York, 1977), pp. 189-244.

[60] J. L. Ericksen, Arch. Ration. Mech. Anal. 73, 99 (1980).

[61] G. P. Parry, Arch. Ration. Mech. Anal. 145, 1 (1998).

[62] I. Folkins, J. Math. Phys. (N.Y.) 32, 1965 (1991).

[63] J. Frenkel and T. Kontorova, Izv. Akad. Nauk SSSR, Ser. Fiz. 1, 137 (1939).

[64] R. Peierls, Proc. Phys. Soc. 52, 34 (1940).

[65] F. R. N. Nabarro, Proc. Phys. Soc. London 59, 256 (1947).

[66] A. S. Kovalev, A. D. Kondratyuk, A. M. Kosevich, and A. I. Landau, Phys. Status Solidi (b) 177, 117 (1993).
[67] A. I. Landau, Phys. Status Solidi (b) 183, 407 (1994).

[68] A. Carpio and L. L. Bonilla, Phys. Rev. Lett. 90, 135502 (2003).

[69] I. Plans, A. Carpio, and L. L. Bonilla, Europhys. Lett. 81, 36001 (2007).

[70] L. L. Bonilla, A. Carpio, and I. Plans, Physica (Amsterdam) 376A, 361 (2007).

[71] P. S. Lomdahl and D. J. Srolovitz, Phys. Rev. Lett. 57, 2702 (1986).

[72] D. Srolovitz and P. Lomdahl, Physica (Amsterdam) 23D, 402 (1986).

[73] O. U. Salman and L. Truskinovsky, Phys. Rev. Lett. 106, 175503 (2011).

[74] A. Minami and A. Onuki, Acta Mater. 55, 2375 (2007).

[75] A. Onuki, Phys. Rev. E 68, 061502 (2003).

[76] A. Carpio and L. L. Bonilla, Phys. Rev. B 71, 134105 (2005).

[77] P.-A. Geslin, B. Appolaire, and A. Finel, Acta Mater. 71, 80 (2014).

[78] I. Fonseca, Arch. Ration. Mech. Anal. 97, 189 (1987)

[79] V. P. Dmitriev, S. B. Rochal, Y. M. Gufan, and P. Toledano, Phys. Rev. Lett. 60, 1958 (1988).

[80] B. Horovitz, R. J. Gooding, and J. A. Krumhansl, Phys. Rev. Lett. 62, 843 (1989).

[81] M. Sanati, A. Saxena, T. Lookman, and R. C. Albers, Phys. Rev. B 63, 224114 (2001).

[82] K. Bhattacharya, S. Conti, G. Zanzotto, and J. Zimmer, Nature (London) 428, 55 (2004).

[83] F. J. Pérez-Reche, L. Truskinovsky, and G. Zanzotto, Continuum Mech. Thermodyn. 21, 17 (2009).

[84] A. Vattré and C. Denoual, J. Mech. Phys. Solids 92, 1 (2016).

[85] S. Conti and G. Zanzotto, Arch. Ration. Mech. Anal. 173, 69 (2004).

[86] G. Puglisi and L. Truskinovsky, J. Mech. Phys. Solids 53, 655 (2005).

[87] A. Mielke and L. Truskinovsky, Arch. Ration. Mech. Anal. 203, 577 (2012).

[88] See Supplemental Material at http://link.aps.org/ supplemental/10.1103/PhysRevLett.123.205501 for details on the energy invariance, the mapping of the configurational space onto the upper complex half plane, the computation of the Klein invariant, and the numerical implementation of the model, and for captions to the Supplemental Movies 1-3 and Supplemental Material Figs. 1 and 2.

[89] P. Engel, Geometric Crystallography: An Axiomatic Introduction to Crystallography (Springer, Dordrecht, 1986).

[90] R. Ogden, Non-Linear Elastic Deformations (Ellis Horwood, Chichester, England, 1984).

[91] Y. Grabovsky and L. Truskinovsky, J. Nonlinear Sci. 24, 1125 (2014).

[92] K. J. Van Vliet, J. Li, T. Zhu, S. Yip, and S. Suresh, Phys. Rev. B 67, 104105 (2003).

[93] R. E. Miller and D. Rodney, J. Mech. Phys. Solids 56, 1203 (2008).

[94] G. P. Parry, Math. Proc. Cambridge Philos. Soc. 80, 189 (1976). 
[95] D. Rodney and J. Bonneville, in Physical Metallurgy, 5th ed., edited by D. E. Laughlin and K. Hono (Elsevier, Oxford, 2014), pp. 1591-1680.

[96] F. Maresca, D. Dragoni, G. Csányi, N. Marzari, and W. A. Curtin, npj Comput. Mater. 4, 69 (2018).

[97] B. Schoeneberg, Elliptic Modular Functions: An Introduction, Grundlehren der mathematischen Wissenschaften, Vol. 203 (Springer Science+Business Media, New York, 2012).

[98] A. van Wijngaarden, in Indagationes Mathematicae (Proceedings) (Elsevier, New York, 1953), Vol. 56, pp. 389-400.

[99] L. Dezerald, L. Ventelon, E. Clouet, C. Denoual, D. Rodney, and F. Willaime, Phys. Rev. B 89, 024104 (2014).

[100] P. Franciosi and A. Zaoui, Int. J. Plast. 7, 295 (1991).

[101] S. Forest, Acta Mater. 46, 3265 (1998).
[102] A. Ask, S. Forest, B. Appolaire, K. Ammar, and O. U. Salman, J. Mech. Phys. Solids 115, 167 (2018).

[103] J. Weiss, W. B. Rhouma, T. Richeton, S. Dechanel, F. Louchet, and L. Truskinovsky, Phys. Rev. Lett. 114, 105504 (2015).

[104] G. Sparks and R. Maaß, Phys. Rev. Mater. 2, 120601 (2018).

[105] J. Rehbein and B. K. Carpenter, Phys. Chem. Chem. Phys. 13, 20906 (2011).

[106] D. Wales, Energy Landscapes: Applications to Clusters, Biomolecules and Glasses (Cambridge University Press, Cambridge, England, 2003).

[107] A. Shtyk, G. Goldstein, and C. Chamon, Phys. Rev. B 95, 035137 (2017).

[108] F. Bociort and M. van Turnhout, in Optical Design and Engineering II, SPIE Proceedings, Vol. 5962 (SPIEInternational Society for Optical Engineering, Bellingham, WA, 2005), p. 59620S. 